Journal of Engineering and Applied Sciences 14 (5): 1601-1607, 2019

ISSN: 1816-949X

(C) Medwell Journals, 2019

\title{
A Handover Scheme for Mobile Ad-Hoc Network Mobility Support
}

\author{
${ }^{1}$ Haider N. Hussain and ${ }^{2}$ Waleed Noori Hussein \\ ${ }^{1}$ Department of Computer Science, \\ ${ }^{2}$ Department of Mathematics, Faculty of Education for Pure Science, University of Basra, Basra, Iraq
}

\begin{abstract}
Mobile Ad-hoc Network (MANET) is a rapidly growing wireless technology with extensive range of applications. MANET routing surfaces many challenges due to their ad-hoc nature and many routing algorithms have been suggested. Reactive routing protocols are preferred due to less control overhead and scalability but they suffer from frequent link breakages due to the high-mobility of the nodes a suitable mobility model should be deployed carefully. To reduce the link breakages and get a stable route we propose a handover scheme to support seamless mobility and continuous connection.
\end{abstract}

Key words: Telecommunications, MANET, PMIPv6, MAG, MN, seamless mobility

\section{INTRODUCTION}

In recent years, there have been big advances in telecommunications equipment. The telecommunication systems have evolved into wireless systems and later mobile systems and new applications have emerged with added performance requirements. However, this rapid growth has led to high handover latencies, especially in mobile devices where there is no permanent infrastructure. In addition, there are new applications (i.e., internet, etc.) and videos that require a low handover latency to support seamless continues connection to run and involve new applications and new telecommunications mechanisms. Furthermore, communications networks mechanisms are being questioned to increase the sensitivity of the transmission rates (transmission performance) for now a days multimedia applications and meet their performance needs. It is expected that future networks architecture will have greater coverage, faster transmission speeds and more reliable guarantees, so that, the applications can include the concept of Future Internet (FI) and Next Generation Wireless Networks (NGWN) which should allow mobile users to be connected anytime and anywhere (Kota, 2011).

One of the leading computer networks that can provide an efficient solution for these types of scenarios (which are already being widely used) are known as Mobile Ad-hoc Networks (MANETs), a type of mobile network which are equipped with wireless devices for computers with dynamic self-optimization self-configuration and self-healing as well as being without a fixed infrastructure. All these devices (smartphones, tablets, mobile clients on laptops, etc.) can act as end users and/or routers, providing alternative routes in a wide range of scenarios. These types of networks can be used in many scenarios such as Flying Ad-hoc Networks (FANETs), Vehicular Ad-hoc Networks (VANETs) and Wireless Sensor Networks (WSN) which can be either static or mobile (Conti and Giordano, 2014; Yan et al., 2013) (Fig. 1).

Traditionally, MANETs have been conceived and deployed with wireless equipment's that use a dedicated ad-hoc radio mode. However, it has been shown to be promising (Gupta and Vatsa, 2011; Hussain et al., 2011; Carvalho et al., 2016) to consider cellular ad-hoc networks where cellular technologies such as WiFi or LTE (infrastructure) are used in a particular way to build ad-hoc networks. In cellular ad-hoc networks, nodes are composed of two radio interfaces as presented in Fig. 2a: one acting as an Access Point (AP), the other one acting as a terminal. Using either WiFi or LTE, this combination allows each node to attach to only one of its neighbors using the terminal interface and to receive many connections from neighboring nodes thanks to the access point radio interface. The network links are thus, built from the attachments that nodes initiate with one another using their terminal interface. A routing protocol makes multi-hop communications possible between connected nodes. Due to nodes mobility, MANET attachments may change over time, leading to a dynamic network topology. Therefore, the handover decisions have to be made with caution, since, they directly impact the global network connectivity.

Off-the-shelf terminal interfaces generally select an access point based on signal strength maximization and pre-configured preferences. However, they do not

Corresponding Author: Haider N. Hussain, Department of Computer Science, Faculty of Education for Pure Science, University of Basra, Basra, Iraq 


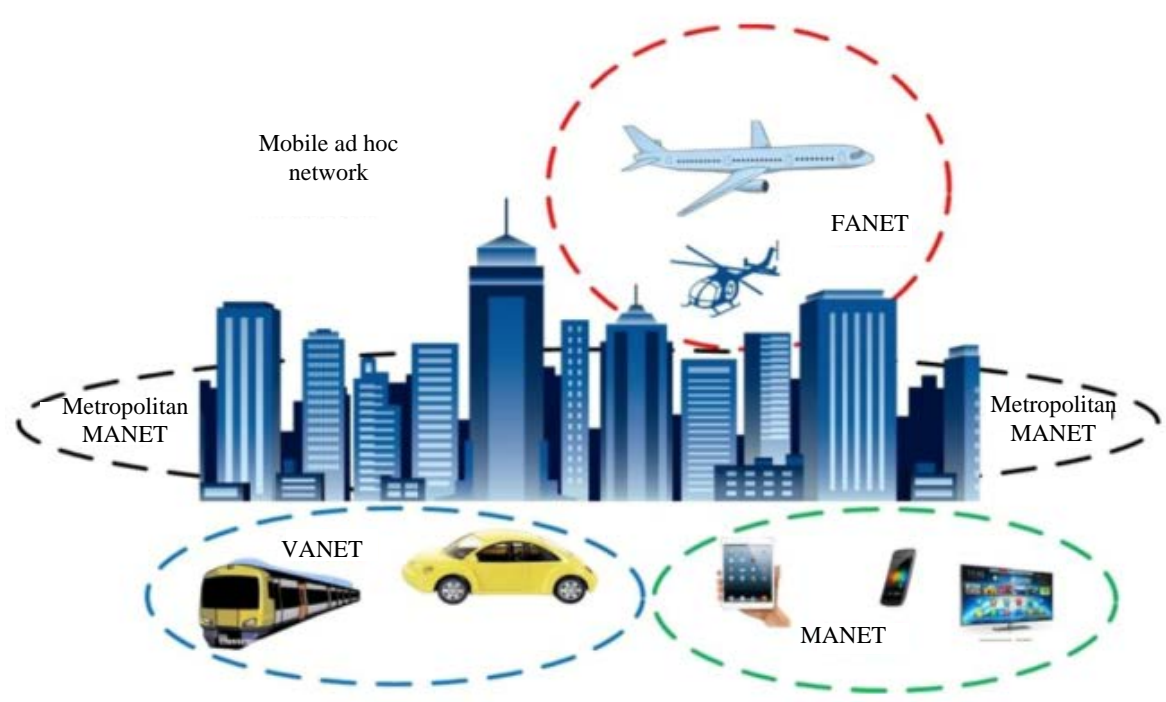

Fig. 1: Mobile Ad hoc Network (MANET) (Carvalho et al., 2016)

(a)

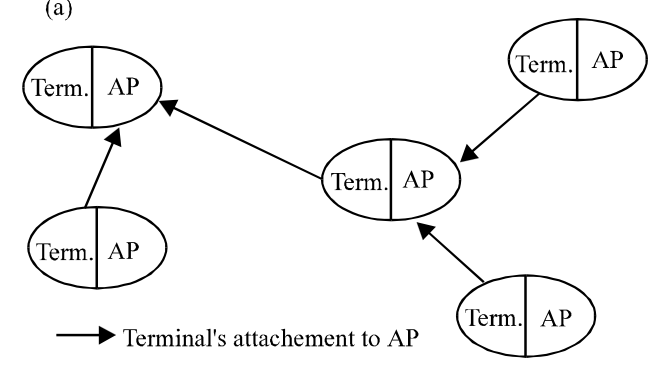

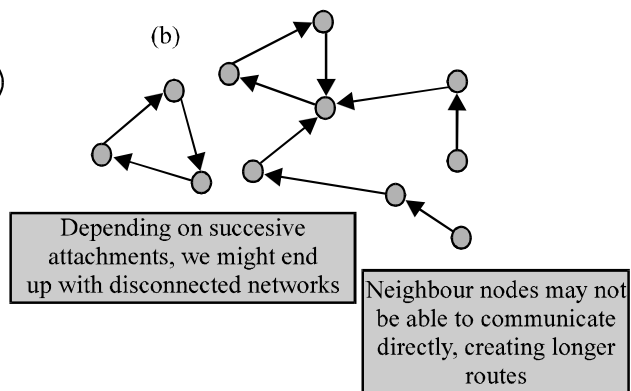

Fig. 2: Cellular ad hoc network, network connectivity: a) Structure of cellular and Ad hoc network and b) Connectivity issues with signal-based attatchment strategies

consider connectivity at the network hierarchy. This may lead to problematic connectivity conditions as shown in Fig. 2b. The network may be disconnected while the radio range would allow full connectivity or may suffer from long routes between nodes that could be closer in the network space.

Literature review: MANETs are maturing area of research and development that has seen many innovations, however, the inherent complexity of the MANET problem domain hasensured that there still remain many challenges to overcome.

Protocols designed for MANETs were originally introduced to support intercommunication between the mobile nodes connected to a MANET but over time they have been augmented to support continuous communication to MANETs MN when roaming between different networks (Attia et al., 2015). If a MN in MANET wishes to roam across heterogeneous networks, each attachment point they connect with must be equipped to support their connection. If the MNs in MANET roams on to a new access network and (in the most straightforward case) is connected to that access network via. asingle point of attachment known as the Foreign Agent (FA) then somethin gmust be done in order to ensure packets can be routed out ofand back in to the MANET. Typically, this could involve using Mobile IP approaches, discussed in the following subsections.

MANET features for mobility: In MANETs, computation involves each Mobile Node ( $\mathrm{MN}$ ) to have multiple addresses (i.e., two addresses) where the $\mathrm{MN}$ uses one address for identification while the other address is used for routing. TCP keep track of all internal session states using IP address of the two endpoints, Fig. 3 shows the 


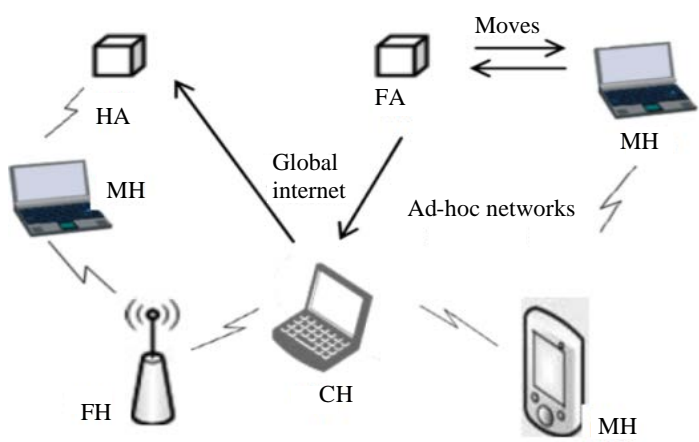

Fig. 3: MANET architectures in MIP networks

MANET architectures in mobile IP Network. While using this IP address to find optimal route based on specified routing algorithm, mobile nodes determine its attachment point either at Home Network (HN) or a Foreign Network (FN) using MIPv6. Both MIPv4 and MIPv6 support node mobility des (Attia et al., 2015) using agent discovery, registration and routing updated with trust implemented in Trusted Carry-Forward (TCF) algorithm.

When the MN detects available access network and get authorized to use $\mathrm{BS} / \mathrm{AP}$, resources/services fixed within coverage area serve as Foreign Host $(\mathrm{FH})$ to the $\mathrm{MN}$ and/or CHs. To inform $\mathrm{MN}$ about its loss of connection, therefore, becomes necessary such that new technique of discovery, registration and routing may be initiated for continued service quality in roaming. Mobility management discussed by Makaya and Pierre (2007) composed of two components of location and handover management. Handover is the mechanism whereby the $\mathrm{MN}$ keeps its connection active when migrating or roaming different coverage area of one access network attachment point to another with similar or different technology.

MIPv6 provide mobility support for IPv6 MN at the network layer (layer 3) (Li et al., 2010). IP extension and technology allow integration of heterogeneous networks into single all-IP based, integrated platform for MIPv6 to create new method of return rout ability procedure to share secrets between $\mathrm{MNs}$ and $\mathrm{CHs}$ as proposedin (Nada, 2006). As the MNroams, away from its home subnet in MANETs, MPv6 implement central data structure cache at $\mathrm{HA}$ for $\mathrm{CH}$ to learn and cache for update of care-of-address at FAs (Ijawoye and Jinadu, 2016).

Proxy mobile IPv6: The internet engineering task force designed proxymobile IPv6 (PMIPv6) to support network-based IP mobility management for MNs without requiring its involvement in any related IP-mobility functions. Mobility management in PMIPv6 is provided to $\mathrm{MN}$ irrespective ofthe presence or absence of Mobile

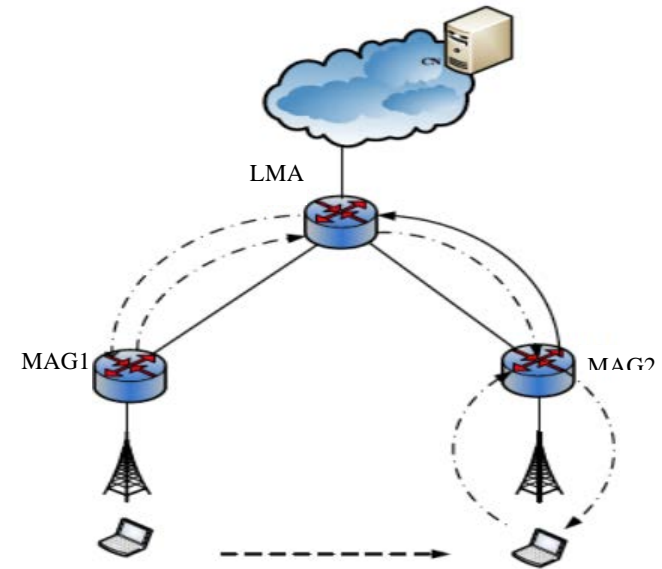

Fig. 4: PMIPv6 network architecture (Hussain et al., 2011)

IPv6 functionality (Boukerche et al., 2011). PMIPv6 extends the signaling of MIPv6 and reuses most of MIPv6 concepts such as HA functionality. In addition, itintroduces two new elements known as Local Mobility Anchor (LMA) and Mobile Access Gateway (MAG) (Attia et al., 2015). Attia et al. (2009) believed that the LMA behaves similar to the HA in MIPv6 in the PMIPv6 domain and that it also introduces additional capabilities required for network-based mobility management. PMIPv6 protocol operation consists of four phases. In the first phase, MAG retrieves the MN's profile using itscurrent identifier. The Binding Update (BU) is the second phase in which the MAG sends a Proxy Binding Update (PUB) request to the LMA in order to register the currentpoint of attachment of the MN. Accordingly a binding cache entry and a tunnel for the MN's home prefix will becreated. The third phase will be the MAG emulating themobile node's home interface on the access interface. Therefore, the MN will always believe it is in the home network. Fourthly, the LMA replies with a Proxy Bind Acknowledge (PBA) message with the MN's HNP. After receiving the Router Advertise (RA) message, the $M N$ configures its IP address by using the contained prefix. Forpacket routing, the LMA is able to route all received packets over the established tunnel to the MAG. The MAG forwards these packets to the MN. Additionally, the MAG will relay all the received packets over the tunnel to the LMA and then they will be routed towards the CN. Figure 4 shows, PMIPv6 network architecture (Nada, 2006).

\section{MATERIALS AND METHODS}

Proposed handover schema: In this study, we present the design of our proposed MANET-PMIPv6 handover schema. With PMIPv6 protocol every MANET sets up a bi-direction tunnel with its HA 
whenever it is able to establish its own direct connection. At the same time all of these nodes also maintain an Ad-hoc communication interface over which they participate in Ad-hoc-on-Demand Vector (AODV) protocol exchange to establish a MANET with another MANET MNs around them. The MANET establish connection with the MAG in PMIPv6 network where the MAG assigns a unique address. In this role, the MANET $\mathrm{MN}$ provides the opportunity for other nodes connected to the MANET toutilize its pre-existing connection with its $\mathrm{HA}$ to forward their packets through the MAG attached with.

Once packets leave the MANET, MAG then employs the proposed technique that ensures any MANET MN can maintain its connection when roaming within different networks. However, MAG does also support the more simplistic case whereby each of the MANET nodes operates as an individual host.

When MANET MN receives the data packets it will use AODV protocol to route the packets to the detonation $M N$. If any $M N$ in MANET movies far from its network the MAG will predicted this movement and will send a PBU message to LMA. When LMA receives the PBU message it will configure a tunnel with the MAG's address and LMA's address as the end points. The data response packet contains the LMA's address. In this way, the MAG can also configure an Ipv6 tunnel to transmit the HNP's related traffic. Furthermore, forinter-domain connection when the LMA receives the PBU message it establishes or refreshes the tunnel with that LMA and the HNP is used as the index of the tunnel.

When the CN sends a packet to the MANET MN, LMA will receive the packet. The LMA then checks the binding state and tunnels the packet to the serving MAG. The MAG maintains the tunnel with the LMA can de-capsulate the packet and tunnel it to the MANET MN address.

MAG operation has been extended as shown in Fig. 5 to support MANET mobility by adding a new extension to the MAG design. Since, the MAG knows the radio link information (i.e., MAC address and channel range of PoAs, etc.). Hence, there will be no need to use the "scanning" mechanism to find neighboring attachment points. In addition, L2 and L 3 are assumed to start simultaneously this is because all the necessary information needed for the handover to start its process will be known as described. In other words with the MAG extension, the MN and the MANET in PMIPv6 domain network entities in particular the MAG in the access routers are informed about the values of the relevant parameters.

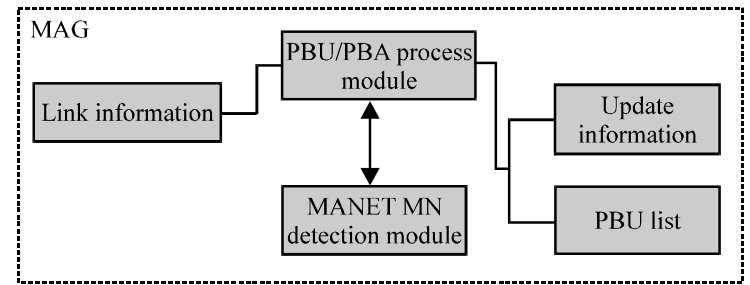

Fig. 5: Proposed MAG extension

(link going up, link going down, etc.,) necessary in handover decision making prior to the actual handover process.

In addition, from PMIPv6 perspective, the main responsibility of MAG is to maintain the information table for tunnels between MAG and LMA (Hussain, 2016). In MAG, the tunnel can have two status values.

Provisional: When the tunnel status is provisional, MAG buffers the data packets received from this tunnel without forwarding them.

Conclusive: When the tunnel status is conclusive, MAG will forward the data packets received from this tunnel to the destination MANET MN. The MAG extension process can be summarized as:

MAG defines one of the MN in the MANET as the MANET MN. When the MAG predicts MANET movement (before detachment/attachment) using the information from the detection model. If the received information contains new signaling information (L2 information), MAG will know that MANET is starting to move to a new domain. In such a case, the MAG will send a fast PBU message to its LMA in order to prevent any loss of packets during the handover process.

This early PBU message will trigger the LMA to estimate MANETs detachment time earlier based on the information retrieved from the PBU message that provides the estimation based on the detection model. When receiving the extended $\mathrm{PBU}$ message LMA will use the analyzed stored information to locate the address stored. A pre-tunnel will be established between both MAGs to support the handover process. After the pre-tunnel establishment, MANET MN will receive a PBU message form the new MAG to configure a new address if the MANET MN is roaming between different LMA network domains.

In order to enhance the handover perdition, we propose an estimation model shown in Fig. 6. The decision to make handover estimation was based on an Estimation Engine (EE). 


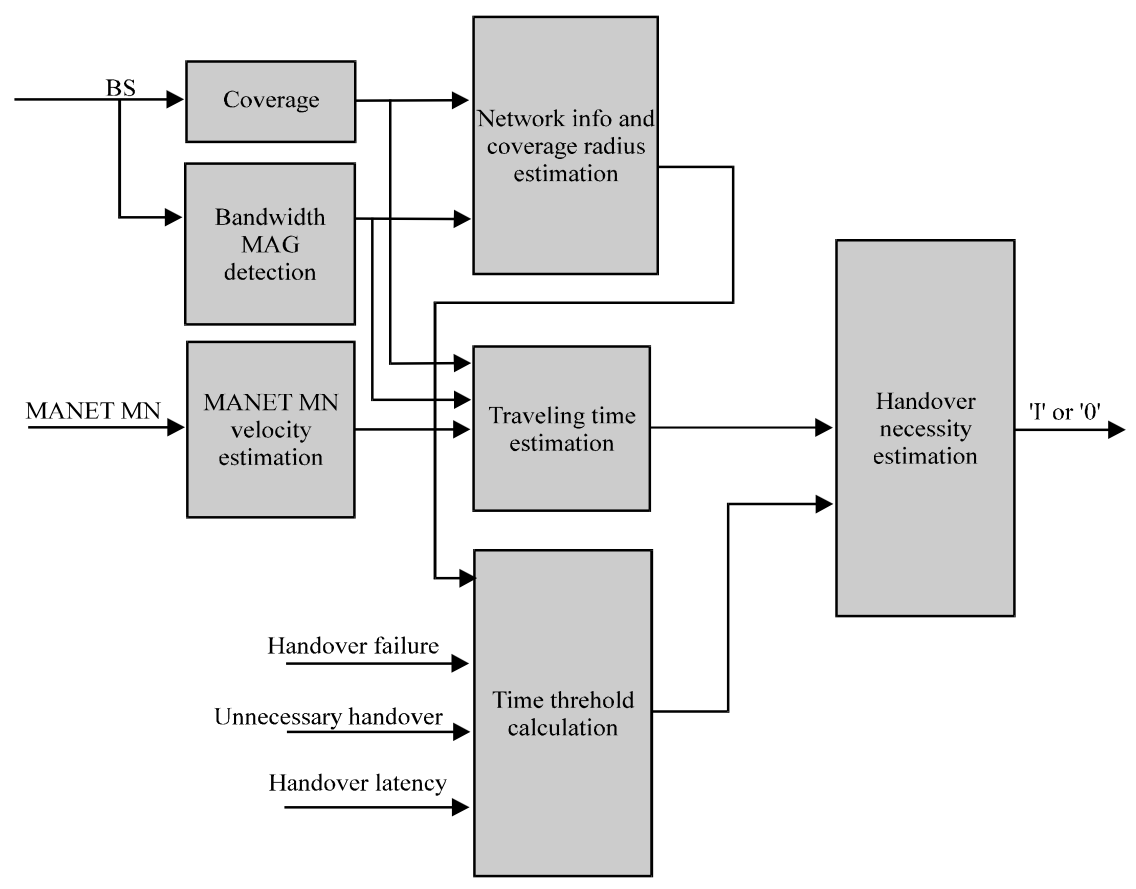

Fig. 6: Proposed MAG estimation model

that was designed to determine the necessity of making the handover between two networks or between two BS or $\mathrm{AP}$ depending on the available information. The information used in the estimation engine was stored within the PBU message that will be used by LMA to predict whether the handover was necessary. A handover Estimation Engine (EE) determines the necessity of making the handover to an available network or Base Station (BS). Various network parameters such as the input and binary output values were considered in the handover estimation process. The inputs included:

- Base Station(BS) bandwidth

- Network radius (communication range)

- MANET MN velocity

Signal: A strategy used by MAG to selects the neighbor PoA with the highest signal quality.

Optimal: A strategy used by LMA that provides the best ad-hoc connectivity. This strategy simulates the traditional ad-hoc interface where nodes can exchange data as soon as they are in radio range (i.e. without the constraint of the cellular mode where one node can only attach to one of its neighbors). Additionally, to handover latency, handover failure and unnecessary handover probability requirement. Algorithm 1 shows the pseudo code for MAG process. In addition, to handover latency, handover failure and unnecessary handover probability requirement.

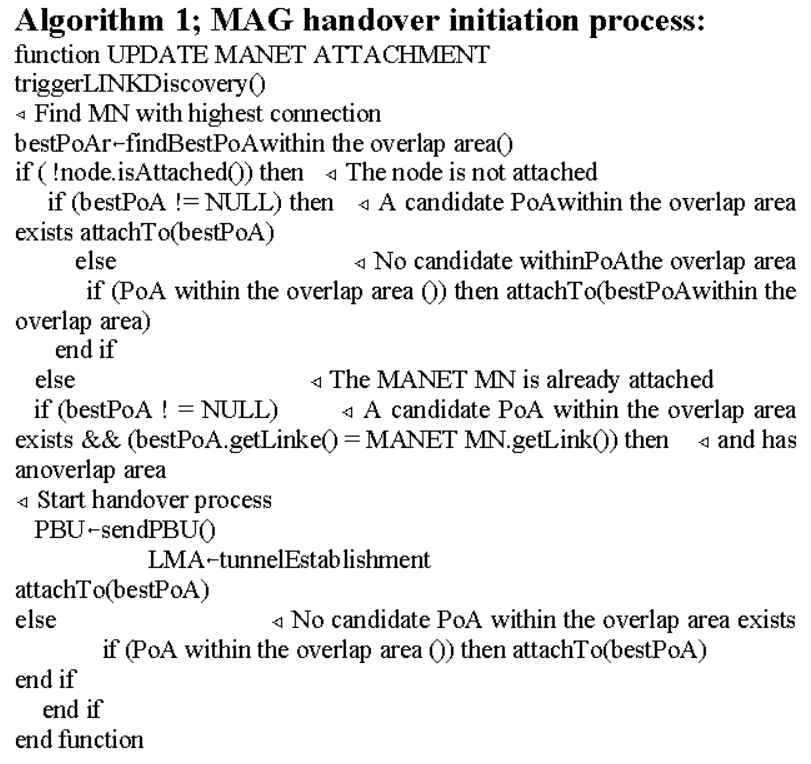

Two thresholds are used to control seamless and continues connection: threshold flow disruptions prevents handovers that would impair too many flows, and threshold path length gain (inside function optimize path length in within the overlap area) precludes handovers that would not generate a sufficient gain on the average path length. Combination of these two thresholds allows swapping between the improvement of connectivity and the negative impact that frequent changes of attachments may have. 


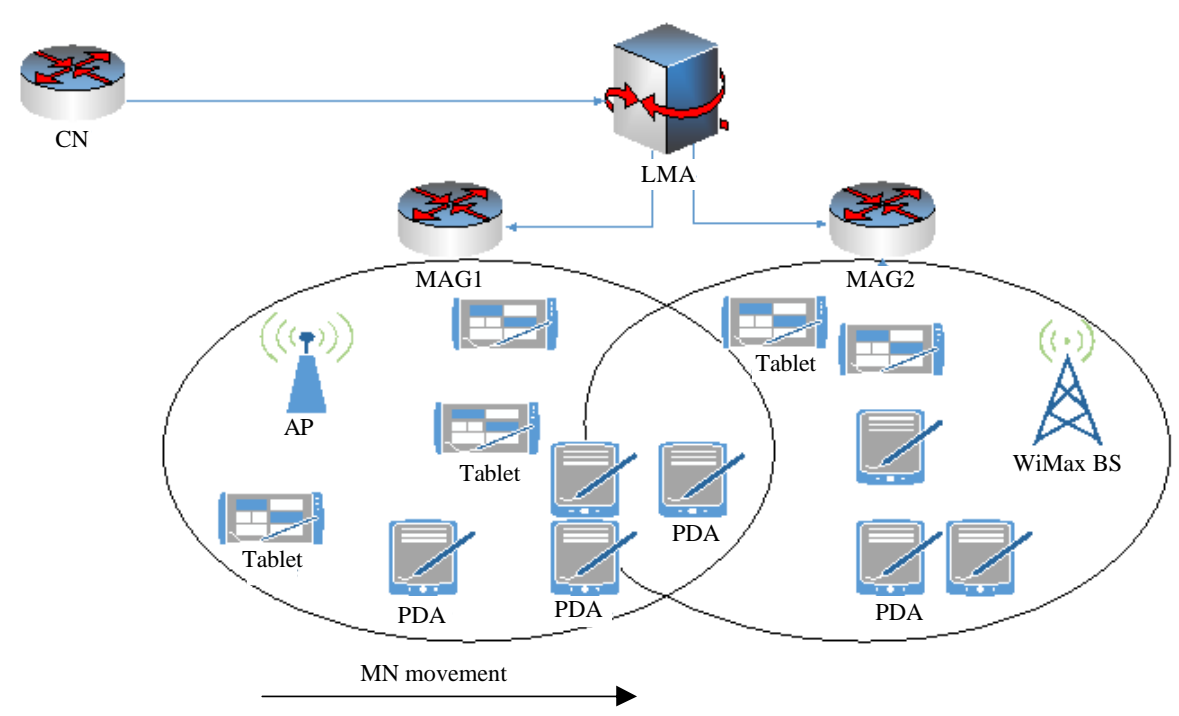

Fig. 7: Proposed MANET-PMIPv6 network architectures

\section{RESULTS AND DISCUSSION}

Network model: In this study, we discuss the proposed MANET-PMIPv6 network architecture shown in Fig. 7. The below components are needed to incorporate for deploying the system.

Road-Side-Unit Controller (RSUC): A cluster of RSUs (e.g., BS) are connected to a RSUC through broadband connections before accessing to the controller. RSUC is hardware flow-enabled and controlled by L-Hanover block controller. Besides the responsibility of forwarding data, RSUCs also store local road system information and perform emergency services. RSUCs are fog devices. Furthermore, cellular Base Station (BS) in our proposed network is not simply carrying voice calls and conveying data it is more sophisticated. The BS is under the L-Hanover block controller, capable of delivering fog services. Similar to RSUC, BS is also local intelligence.

MAG is the mainly evolved component in PMIPv6-Fog scheme in comparison with PMIPv6. The two major changes in MAG comparing to MAG in PMIPv6 are: It supports the HardWare (HW) flow table, MIIS, PMIPv6 control signaling functionalities of MAG have been offloaded to the L-Hanover block controller. The main functionalities of MAG are briefly described as.

Link state monitoring: $M A G$ monitors the link state of $\mathrm{MN}$ in MANET attached to it by monitoring the HW flow table, MIIS information value of the received frames/packets. These link state values are maintained as an exponential moving average and when signal drops below a threshold MAG reports this event to the L-Hanover block controller by sending MNs
MANET-ID and link state value. The MAG also snap shots the link state of MNs in MANET which are in its vicinity but are not attached to it by utilizing the monitor mode of the IEEE 802 Standard. If the link state of any such $M N$ is above a certain threshold then it is reports to the L-Hanover block controller by sending the MNs MANET-ID and snap shot link state value (Bhute et al., 2016).

\section{CONCLUSION}

In this research, we proposea theoretical solution to improve the handover process in mobile Ad-hoc networks. The proposal is based upon a MANET-PMIPv6 mobility scheme that reduces the handover process by reducing the signaling time needed for the handover to complete its process as compared to single node mobility scheme. Thus, network load is reduced.

To further improve the performance of handover scheme we consider anestimation model-based handover mechanism that provides efficient usage criteria among various network situations in the disaster area. The main idea is at adapt a handover approach for MANET by having reactive and proactive handover mechanism incorporated with the policy-based architecture that greatly improves the efficiency and network extendibility and could assure better QoS values for the user.

We are currently working to develop a mathematical and simulation model for our proposed handover scheme. Furthermore, the proposed scheme will be tested and compared with other MANET routing protocols to evaluate the proper protocol that will be efficient to use with MANET-PMIPv6 mobility model. 


\section{REFERENCES}

Attia, R., R. Rizk and H.A. Ali, 2015. Internet connectivity for mobile ad hoc network: A survey based study. Wireless Networks, 21: 2369-2394.

Attia, R., R. Rizk and M. Mariee, 2009. A hybrid multi-path ant $\mathrm{Q} O S$ routing algorithm for MANETs. Proceedings of the IEEE/IFIP International Conference on Wireless and Optimal Communications Networks, April 28-30, 2009, Cairo, Egypt, pp: 1-5.

Bhute, H., G.T. Chavan and A. Bhute, 2016. Analysis of location management schemes for MANET using synthetic mobility models. Networking Internet Archit., 2016: 1-7.

Boukerche, A., B. Turgut, N. Aydin, M.Z. Ahmad, L. Boloni and D. Turgut, 2011. Routing protocols in Ad Hoc networks: A survey. Comput. Networks, 55: 3032-3080.

Carvalho, T.C.D., J.J.J. Ferreira and R.L. Frances, 2016. Reliable energy-efficient multilayer mechanism with realistic battery model and QoE support in wireless MANETs. J. Microwaves Optoelectron. Electromagn. Appl., 15: 30-48.

Conti, M. and S. Giordano, 2014. Mobile ad hoc networking: Milestones, challenges and new research directions. IEEE Commun. Mag., 52: 85-96.

Gupta, A. and A.K. Vatsa, 2011. Policy based fast handoff mechanism for MANET. World Comput. Sci. Inf. Technol. J., 1: 339-345.

Hussain, H.N., 2016. A PMIPv6-Fog scheme for vehicular mobility support. J. Comput. Eng., 18: 61-6610.9790/0661-1806056166.
Hussain, H.N., K.A. Bakar and S. Salleh, 2011. A novel intra-domain continues handover solution for inter-domain PMIPv6 based vehicular network. Intl. J. Adv. Comput. Sci. Appl., 2: 12-18.

Ijawoye, R.O. and O.T. Jinadu, 2016. Multi-Access support with hybrid handover proposed for MANET users and IEEE 802.22 Networks. Intl. J. Eng. Innovative Technol., 5: 1-6.

Kota, S.L., 2011. Hybrid-integrated networking for NGN services. Proceedings of the 2011 2nd International Conference on Wireless Communication, Vehicular Technology, Information Theory and Aerospace and Electronic Systems Technology (Wireless VITAE), February 28-March 3, 2011, IEEE, Chennai, India, ISBN:978-1-4577-0787-2, pp: $1-6$.

Li, Q., J. Tatuya and K. Shima, 2010. Mobile IPv6 Networks. Morgan Kaufmann Publishers, Burlington, Massachusetts, USA.,.

Makaya, C. and S. Pierre, 2007. Trends and Challenges for Mobility Management in IP-Based Next Generation Wireless Networks. In: Wireless Communications Research Trends, Lee, T.S. (Ed.). Nova Science Publishers, Hauppauge, New York, USA., ISBN:9781600216749, pp: 83-122.

Nada, F.A., 2006. On using mobile IP protocols. J. Comput. Sci., 2: 211-217.

Yan, R., H. Su and Y. Qian, 2013. Energy-aware sensor node design with its application in wireless sensor networks. IEEE Trans. Instrum. Measure., 62: 1183-1191. 